\title{
Risk of Proximal Femoral Nail Antirotation Implant Failure Upon Different Lateral Femoral Wall Thickness in Intertrochanteric Fracture :A Finite Element Analysis.
}

\section{Liqin Zheng}

Guangzhou University of Chinese Medicine

\section{Duo Wai-Chi Wong}

Hong Kong Polytechnic University

\section{Xinming Chen}

Wuhan University Renmin Hospital

\section{Yuanzhuang Chen}

Jiangmen Central Hospital

Pengfei Li ( $\sim$ lpfdedicated@yeah.net)

Jiangmen Central Hospital https://orcid.org/0000-0002-3222-9112

\section{Research article}

Keywords: fracture mechanics, finite element analysis, Proximal Femoral Nail Antirotation

Posted Date: September 18th, 2020

DOI: https://doi.org/10.21203/rs.3.rs-73487/v1

License: (c) (1) This work is licensed under a Creative Commons Attribution 4.0 International License.

Read Full License 


\section{Abstract}

\section{Purpose}

PFNA has been commonly used to treat intertrochanteric fractures, despite the risk of implant failure. The integrity of the femur could influence the risk of implant failure. This study aims to evaluate the influence of lateral femoral wall thickness on potential implant failure using a computational modeling approach.

\section{Methods}

Finite element model of the hip was reconstructed from the Computed Tomography of a female patient. Five intertrochanteric fracture models at different lateral femoral wall thickness $(T 1=27.6 \mathrm{~mm}, \mathrm{~T} 2=25.4$ $\mathrm{mm}, \mathrm{T} 3=23.4 \mathrm{~mm}, \mathrm{~T} 4=21.4 \mathrm{~mm}$, and T5 $=19.3 \mathrm{~mm}$ ) were created and fixed with PFNA. A critical loading condition was simulated that mimicked a high loading scenario during walking. The implant failure condition, stress and displacement of the PFNA implant and fracture femur were predicted for analysis.

Results

Implant failure of PNFA occurred at the sides of the proximal nail canal especially for the thinner wall models (T4 and T5). The maximum von Mises stress of the nail for T4 changed abruptly to $298.1 \mathrm{MPa}$. However, thinner wall decreased the displacement of the PFNA implant. There was approximately opponent trend of stress and displacement on proximal and distal fragments with decreasing thickness possibly due to the adaptation after failure.

Conclusion

A thinner wall increased the risk of PFNA implant failure. Our prediction showed that complete failure occurred when the thickness was $21.4 \mathrm{~mm}$ which was close to the value suggested to determine the stability type.

\section{Introduction}

Proximal Femoral Antirotation Intramedullary Nail (PFNA) is considered as one of the standard internal fixation approaches for intertrochanteric fracture. The approach has high cut-out resistance and biomechanical stability that retard rotation and varus collapse and was proven to relieve pain and facilitate early mobilization [1]. Besides, PFNA enables easy manipulation, minimizes soft tissue dissection and thus facilitates shorter operation time, reduced healing time, and hospital stay $[1,2]$. Nonetheless, PFNA is not well-indicated for patients with severe osteoporosis or complicated fractures[2]. Complications were reported in about $8 \%$ of the patients after PFNA such as implant failure/breakage and fixation failure requiring reoperation [3]. Figure 1 demonstrates a case of complication with PFNA nail fracture. 
The biomechanics of PFNA plays an important role in the occurrence of implant failure [3]. The pattern of implant failures was similar and predictable that was associated with the excessive sliding and toggling of the femoral neck screw [3]. The intramedullary nails of the PFNA could sustain high forces but the spiral blade may not maintain the femoral shaft adequately [3]. Withal, we believe that the integrity of the femur and stability of the fracture influence the risk of implant failure in PFNA. In fact, the surgical process of PFNA involve reaming the medullary cavity that impairs the load-carrying capacity and stability of the femur. This may result in high stress concentration on the implant and thus failure when in contrary the implant stress gradually declined towards the bone healing process [4].

Lateral femoral wall thickness reflects the integrity and stability of the intertrochanteric fracture condition [5]. It was defined as the distance of a line started from a point 3-cm inferior to the innominate tubercle of the greater trochanter, angled at $135 \rrbracket$ from the upward direction, and headed towards the fracture line on the coronal plane [6], as illustrated in Figure 2. The Orthopaedic Trauma Association Committee for Coding and Classification (AO/OTA) ranked the fracture with higher severity when the lateral femoral wall thickness was more than $20.5 \mathrm{~mm}$ in which the threshold value was justified by the rate of secondary fracture and complications [5]. Sensitivity analysis of the lateral femoral wall thickness on the

biomechanical environment of the fracture and fixation could reveal the mechanism of the implant failure but difficult to conduct by clinical or cadaveric studies. To this end, we developed a three-dimensional FE model of a femur and simulated with intertrochanteric fractures at different lateral femoral wall thickness followed by the PFNA procedure under a critical loading condition. The objective of this study is to evaluate the influence of lateral femoral wall thickness on implant failure of the PFNA. The implant failure/fracture can be simulated and realized by the element erosion function of the FE analysis which was rarely used to predict the biomechanical performance of PFNA and intertrochanteric fracture.

\section{Materials And Methods}

\section{Patient Information}

The female patient was admitted to the hospital on 14 May 2019 because of hip pain on the left side, and x-ray revealed femoral fracture. She was 71 years old, $165 \mathrm{~cm}$ tall and weighed $58 \mathrm{~kg}$. The patient was notified and consented to contribute her medical records and images for the research. The study was approved by the ethical committee of the hospital.

\section{Model Reconstruction and Mesh Creation}

Computed Tomography (CT) of the right hip was acquired using CT (Siemens, Munich, Germany) at 2 $\mathrm{mm}$ slice interval. The CT data in DICOM format was imported into the software Mimics (Materialise, Leuven, Belgium) for processing, in which the femoral bone were segmented based on their grayscale at a threshold from 226 to 3017. Geomagic Studio (Geomagic, 3D Systems, Rock Hill, USA) was used to generate surfaces and reconstructed the three-dimensional geometry of the femur. The Hypermesh (Altair, Troy, USA) was used to tetrahedral meshing and developed the femur into a composited model containing cortical layer and trabecular core by organizing the elements. The size of the mesh was $2 \mathrm{~mm}$. 
Five intertrochanteric fracture models $(\mathrm{T} 1, \mathrm{~T} 2, \mathrm{~T} 3, \mathrm{~T} 4$, and $\mathrm{T} 5)$ at different lateral femoral wall thickness were created on the reconstructed femur of the patient based on the classification of 31A1.3 fractures. Specifications of the five models are listed in Table 1. The procedure to create the fracture line was as followed: Firstly, the first osteotomy line was created by linking the apex of the greater trochanter and the base of the lesser trochanter on the frontal plane, which corresponded to the first fracture model (T1) with a measured lateral femoral wall thickness of $27.6 \mathrm{~mm}$. Secondly, the second osteotomy line was created by rotating the first osteotomy line around the base of the lesser trochanter at $5 \nabla$ anticlockwise on the frontal plane for the second fracture model (T2). Lastly, the other osteotomy lines and fracture models

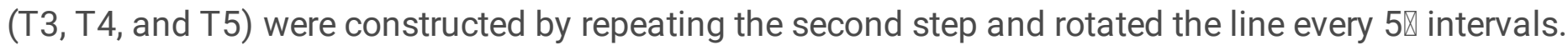
The five intertrochanteric fracture models are shown in Figure 2.

Table 1. The lateral femoral wall thickness and osteotomy line angle of the five fracture models.

\begin{tabular}{|lll|}
\hline Fracture Model & Lateral Femoral Wall Thickness $(\mathrm{mm})$ & Osteotomy Line Angle $(\square)$ \\
\hline T1 & 27.6 & 60 \\
\hline T2 & 25.4 & 55 \\
\hline T3 & 23.4 & 50 \\
\hline T4 & 21.4 & 45 \\
\hline T5 & 19.3 & 40 \\
\hline
\end{tabular}

Regarding the implant, the three-dimensional geometry of PFNA (Dabo Medical Devices Co., Ltd.Xiamen, China) was built in SolidWorks (Solidworks Inc., Dassault Systèmes, Vélizy-Villacoublay, France). The PFNA implant consisted of a nail, a spiral blade, and a locking screw. The length of the PFNA nail was $200 \mathrm{~mm}$ with a neck stem angle of $130^{\circ}$. The proximal and distal diameters of the nail were $16 \mathrm{~mm}$ and $10 \mathrm{~mm}$, respectively. The length of the spiral blade was $107 \mathrm{~mm}$ with a diameter of $10 \mathrm{~mm}$. The PNFA implant model was assembled with the intertrochanteric fracture models according to the PNFA surgical guidelines. In brief, the Tip Apex Distance (TAD) on both the anteroposterior and lateral views, shall be not more than $27 \mathrm{~mm}[7]$.

\section{Material Properties}

The material properties are listed in Table 2. The Elastic modulus of the cancellous bone and cortical bone were assigned based on the constitutive equation between apparent density and elastic modulus. Material failure simulation was enabled for the bone and implant. Elastoplastic material properties were assigned including the yielding stress, failure strain, and the strain rate effects (Cowper-Symonds model) [8]. Material fracture or failure could be resembled by the propagation of element deletion in the FE simulation.

Table 2. Material properties of the trabecular, cortical bone, and PFNA implant used in the FE model. 


\begin{tabular}{|llll|}
\hline Parameters & Trabecular bone & Cortical Bone & Titanium alloy (PFNA) \\
\hline Apparent density $\left(\mathrm{g} / \mathrm{cm}^{3}\right)$ & 0.589 & 1.525 & $4.43[9]$ \\
\hline Elastic modulus $(\mathrm{GPa})$ & 0.496 & 8.318 & $110[10]$ \\
\hline Poisson's ratio & 0.30 & 0.30 & $0.31[11]$ \\
\hline Tangent modulus $(\mathrm{MPa})$ & 49.6 & 831.8 & $1,592[11]$ \\
\hline Yield stress (MPa) & $17.45[12]$ & $109[12]$ & 250 \\
\hline Hardening parameter & $0.10[8]$ & $0.10[8]$ & $0.12[13]$ \\
\hline Failure strain(\%) & $0.7[12]$ & $0.9[14]$ & $0.2[15]$ \\
\hline Cowper-Symonds model parameter & $\mathrm{C:} 2.5$ & $\mathrm{C:} 2.5$ & $\mathrm{C:}: 80000$ \\
& $\mathrm{P}: 7.0[8]$ & $\mathrm{P}: 7.0[8]$ & $\mathrm{P}: 1.1[16]$ \\
\hline
\end{tabular}

\section{Boundary and Loading Conditions}

We simulated a critical loading case resembling the suggested high loading condition during walking [17]. The femoral head was loaded $2,100 \mathrm{~N}$ at $10^{\circ}$ lateral to the inferior axis on the frontal plane and $9^{\circ}$ posterior to the inferior axis on the sagittal plane [17], as demonstrated in Figure 3. The distal section of the femur was fully fixed throughout the simulation.

The coefficients of friction between the implant interfaces, between the fractured bone fragments, and between the implant and the bone were 0.23 [18], 0.46 [18], and 0.3 [19] respectively.

\section{Model Output and Data Analysis}

FE analysis was conducted using LS-DYNA software (LSTC, Livermore, CA, USA). The von Mises stress and displacement of the bone and PFNA, as well as the breakage condition of the implant among the 5 intertrochanteric fracture models with different lateral femoral wall thickness were extracted and analyzed.

\section{Results}

\section{Failure Condition of the PNFA Implant}

As shown in Figure 4, implant failure of PNFA occurred at the sides of the proximal nail canal where the spiral blade passed through. For fractures with a thinner wall thickness (i.e. T4 and T5), the nail was completed broken and detached. For fractures with a higher thickness (T1, T2, and T3), the stress of the material just reached the critical point of failure. A crack or partial defect was manifested on the sides of the canal. Table 3 compares the maximum von Mises stress of the bone fragments and implants among conditions. 
Table 3. Maximum von Mises stress of bone fragments and PNFA implant components (MPa).

\begin{tabular}{|llllll|}
\hline Condition & $\begin{array}{l}\text { Femoral proximal } \\
\text { fragment }\end{array}$ & $\begin{array}{l}\text { Femoral distal } \\
\text { fragment }\end{array}$ & $\begin{array}{l}\text { Spiral } \\
\text { blade }\end{array}$ & Nail & $\begin{array}{l}\text { Locking } \\
\text { screw }\end{array}$ \\
\hline T1 & 243.0 & 211.0 & 333.3 & 247.2 & 248.2 \\
\hline T2 & 173.7 & 191.9 & 286.2 & 244.2 & 287.7 \\
\hline T3 & 165.8 & 196.9 & 302.4 & 252.2 & 279.3 \\
\hline T4 & 226.5 & 203.4 & 174.4 & 298.1 & 335.5 \\
\hline T5 & 219.4 & 208.5 & 172.2 & 254.1 & 332.2 \\
\hline
\end{tabular}

Stress and Displacement of PFNA implant

The von Mises stress was generally concentrated at the contact interfaces between implant components. For the PNFA nail, stress was concentrated at the proximal and distal canals which passed through with the spiral blade and locking screw, respectively (Figure 5). The stress of the spiral blade decreased with increasing thickness of the lateral femoral wall from 333.3 MPa in T1 to $172.2 \mathrm{MPa}$ in T5 (Table 3). The maximum axial displacement and resultant displacement appeared at the tip of the spiral blade. The maximum resultant displacement decreased from $3.653 \mathrm{~mm}$ to $3.015 \mathrm{~mm}$ when the lateral femoral wall thickness decreased from $\mathrm{T} 1$ to $\mathrm{T} 5$ condition.

Table 4. The maximum axial and resultant displacement of PFNA components ( $\mathrm{mm})$

\begin{tabular}{|lllllll|}
\hline Components & Parameters & $\mathrm{T} 1$ & $\mathrm{~T} 2$ & $\mathrm{~T}$ & $\mathrm{~T} 4$ & $\mathrm{~T}$ \\
\hline Spiral blade & $\mathrm{X}$ & 1.757 & 1.678 & 1.579 & 1.520 & 1.416 \\
\cline { 2 - 7 } & $\mathrm{Y}$ & 0.557 & 0.635 & 0.456 & 0.497 & 0.446 \\
& $\mathrm{Z}$ & -3.442 & -3.174 & -3.076 & -2.999 & -2.921 \\
\hline Nail & $\mathrm{R}$ & 3.653 & 3.316 & 3.303 & 3.137 & 3.015 \\
& $\mathrm{X}$ & -0.638 & -0.820 & -0.941 & -1.013 & -1.175 \\
& $\mathrm{Y}$ & 0.256 & 0.285 & 0.293 & 0.341 & 0.525 \\
& $\mathrm{Z}$ & -0.545 & -0.648 & -0.877 & -0.690 & -1.109 \\
\hline Locking screw & $\mathrm{R}$ & 0.877 & 1.036 & 1.193 & 1.218 & 1.699 \\
& $\mathrm{X}$ & -0.054 & -0.051 & -0.071 & -0.090 & -0.107 \\
\cline { 2 - 7 } & $\mathrm{Y}$ & 0.052 & 0.051 & 0.057 & 0.067 & 0.076 \\
& $\mathrm{Z}$ & -0.173 & -0.182 & -0.193 & -0.235 & -0.239 \\
& $\mathrm{R}$ & 0.175 & 0.184 & 0.196 & 0.237 & 0.244 \\
\hline
\end{tabular}


$\mathrm{X}(+)$ :medial direction; $\mathrm{Y}(+)$ :posterior direction; $\mathrm{Z(+)}$ superior direction; $\mathrm{R}$ : resultant

Stress concentration appeared at the middle portion of the locking screw, whereas the maximum displacement was located at the tip of the screw. When the lateral femoral wall thickness decreased from $\mathrm{T} 1$ to $\mathrm{T} 5$, the maximum von Mises stress, and the resultant displacement increased from 248.2 MPa to 332.2 MPa, and from $0.175 \mathrm{~mm}$ to $0.244 \mathrm{~mm}$, respectively (Table 3 and Table 4). Stress concentration appeared around the proximal nail canal throughout all simulated wall thickness conditions, but the maximum stress over overshot at T4. The maximum resultant displacement was presented at the proximal end of the nail before breakage and around the proximal nail canal after breakage (Figure 6). The maximum resultant displacement increased from $0.877 \mathrm{~mm}$ to $1.699 \mathrm{~mm}$ when the lateral femoral wall decreased from T1 to T5 (Table 4).

\section{Stress and Displacementof Fracture Femur}

For the proximal femoral fragment (i.e. femoral head and neck), the location of the maximum von Mises stress depended on the lateral femoral wall thickness. For T1 and T2, the lateral femoral wall thickness corresponded to a fracture line inside the entry point such that the stress was concentrated at the interoinferior cortex of the bone (Figure 7). On the contrary, a fracture line outside the entry point (i.e. the cannulation axis along the PNFA nail) in condition T3,T4 and T5 resulted in a stress concentration appeared around the entry point and the interoinferior femoral neck. The maximum von Mises stress gradually decreased from T1 to T3, and switched to increase from T4 to T5 (Table 3). The maximum resultant displacement of the femur model located at the femoral head. The value decreased from 3.698 $\mathrm{mm}$ to $3.185 \mathrm{~mm}$ when the lateral femoral wall thickness decreased from T1 to T5 as shown in Table 5 .

Table 5. The maximum axial and resultant displacement of the fractured femoral fragments $(\mathrm{mm})$

\begin{tabular}{|lllllll|}
\hline Components & Parameters & $\mathrm{T} 1$ & $\mathrm{~T} 2$ & $\mathrm{~T} 3$ & $\mathrm{~T} 4$ & $\mathrm{~T}$ \\
\hline Proximal fragment & $\mathrm{X}$ & -1.983 & -1.927 & -1.709 & -1.619 & -1.588 \\
& $\mathrm{Y}$ & 0.656 & 0.569 & 0.536 & 0.53 & 0.648 \\
& $\mathrm{Z}$ & -3.649 & -3.461 & -3.301 & -3.203 & -3.116 \\
\cline { 2 - 6 } & $\mathrm{R}$ & 3.698 & 3.526 & 3.374 & 3.273 & 3.185 \\
\hline Distal fragment & $\mathrm{X}$ & -0.552 & -0.691 & -0.727 & -0.808 & -0.842 \\
& $\mathrm{Y}$ & 0.204 & 0.222 & 0.219 & 0.195 & 0.226 \\
& $\mathrm{Z}$ & -0.416 & -0.430 & -0.353 & -0.335 & -0.32 \\
& $\mathrm{R}$ & 0.608 & 0.804 & 0.776 & 0.85 & 0.891 \\
\hline
\end{tabular}

$\mathrm{X}(+)$ :medial direction; $\mathrm{Y}(+)$ :posterior direction; $\mathrm{Z}(+)$ superior direction; $\mathrm{R}$ : resultant 


\section{Discussion}

Stress concentration appeared at the implant interfaces of the PFNA, which was consistent with existing research [20]. The stress of the PFNA components was consistently higher than that of the femur bone in all lateral femoral wall thickness conditions. The stiffer implant took up the load from the femur resulting in stress shielding. As the thickness of the lateral wall decreased, the PFNA stress transferred distally from the spiral blade to the locking screw which was also dependent on whether the upper end of the fracture line crossed the PFNA nail. A thick lateral femoral wall manifested a sloped fracture line that generated a high shear force on the spiral blade. In other words, a vertical fracture line could may induce a shear force on the PFNA as large as the applied load on the femoral head. A Medical Sustainable Nail presented similar construct but featured two nails (cephalic nail and sustainable nail) on the nail shaft which may explain the stronger support in the vertical direction for the fixation of unstable fractures [17].

The intactness of the greater trochanter may reduce the risk of implant failure. The AO/OTA classification suggested that a cut-off femoral lateral wall thickness of $20.5 \mathrm{~mm}$ determines the intactness and completeness of the lateral wall, and thus the overall stability of the fracture [5]. Nearly a quarter of femoral intertrochanteric fracture with incomplete lateral wall required a revision surgery in which nearly three quarters of the fracture happened during the primary surgery [21]. Ignoring incomplete lateral bone insufficiency before the operation or recurring fractures on the lateral wall after surgery may result in shortened fractures, delayed healing, and even revision [22].

When the thickness of the lateral wall gradually decreased until the top of the great trochanter was intact, the bone formed an interlocking structure with the PFNA nail that reduced the tensile stress on the exteroir superior proximal femur, proximal fragment of the femur and the spiral blade. The displacement of the proximal fracture and spiral blade also decreases. The "interlock" effect formed by the top of the great trochanteric and the main nail is reflected in both the coronal and sagittal planes. When the upper end of the fracture line was outside the entry point, the effects of varus and rotation after PFNA internal fixation were suppressed which was similar to another implant design, InterTan that consisted of two integrated proximal screws to control the fragment alignment [23].

The medial wall, lateral wall and the intramedullary fixation resembled a support structure with 3 pillars. Compared to the lateral wall, the medial wall may contribute more to the structural strength of the proximal femur. In addition, it was reported that cortical bone thickness of the femur was positively correlated to its strength. Although the condition of the lateral wall could be an important risk factor to the fracture, the exact mechanism are still unclear [6].

The significance of this study lay in its potential to challenge the dose-effect relationship between the femoral lateral wall thickness and the risk of PFNA implant failure in terms of the stress and displacement of the bone and implant from a biomechanical point of perspective. At T4 and T5 conditions, the prediction showed that the fracture was triggered in the PFNA nail where the location of the fracture was the same as that reported in the fatigue test [17]. This suggested that the femoral lateral wall thickness at T4-T5 $(21.368 \mathrm{~mm}$ to $19.342 \mathrm{~mm})$ may be both the critical value of the PFNA breakage. 
This theoretical analysis using finite element analysis produced a more quantitative and mechanistic information to supplement the AO/OTA fracture classification that determined the stability type at a wall thickness of $20.5 \mathrm{~mm}$ [5]. Based on our findings and discussion, we believed that PNFA implant failure could be initiated around the PFNA nail canal and the locking screw because of the high and concentrated stress.

There were some shortcomings in this study. Firstly, the material property of the femur was assumed isotropic that may influence the pattern of implant failure and fracture in the FE prediction [24]. An anisotropic property of bone including the elastic modulus and the yielding stress are required. Secondly, this study resembled a static critical loading condition since the simulation of fatigue failure required more complicated input in boundary and loading conditions. Therefore, the outcome of this study may deviate from that of the experiments in previous studies. A cadaveric biomechanical test that PFNA under fatigue condition is still advocated to support these findings.

\section{Declarations}

\section{Acknowledgements}

The study was supported by grants from Jiangmen Medical and Health Technology Project(NO 2019E008).

Funding. The study was supported by grants from Jiangmen Medical and Health Technology Project(NO 2019E008)

Conflicts of interest. Not Applicable

Ethical Approval. The study was approved by the Ethical Committee of the Jiangmen Central Hospital (reference number: )

Availability of data: Available upon request

\section{References}

1. Mereddy P, Kamath S, Ramakrishnan M, Malik H, Donnachie N (2009) The AO/ASIF proximal femoral nail antirotation (PFNA): a new design for the treatment of unstable proximal femoral fractures. Injury 40 (4):428-432

2. Jiang X, Wang Y, Ma X, Ma J, Wang C, Zhang C, Han Z, Sun L, Lu B (2016) Proximal femoral nail antirotation versus reverse less invasive stabilization system-distal femur for treating proximal femoral fractures: a meta-analysis. Medicine 95 (14)

3. Makki D, Matar HE, Jacob N, Lipscombe S, Gudena R (2015) Comparison of the reconstruction trochanteric antigrade nail (TAN) with the proximal femoral nail antirotation (PFNA) in the management of reverse oblique intertrochanteric hip fractures. Injury 46 (12):2389-2393 
4. Sitthiseripratip K, Van Oosterwyck H, Vander Sloten J, Mahaisavariya B, Bohez E, Suwanprateeb J, Van Audekercke R, Oris P (2003) Finite element study of trochanteric gamma nail for trochanteric fracture. Medical engineering \& physics 25 (2):99-106

5. Meinberg EG, Agel J, Roberts CS, Karam MD, Kellam JF (2018) Fracture and dislocation classification compendium-2018. Journal of orthopaedic trauma 32:S1-S10

6. Hsu C, Shih C, Wang C, Huang K (2013) Lateral femoral wall thickness: A reliable predictor of postoperative lateral wall fracture in intertrochanteric fractures. The bone \& joint journal 95 (8):1134-1138

7. Yam M, Chawla A, Kwek E (2017) Rewriting the tip apex distance for the proximal femoral nail antirotation. Injury 48 (8):1843-1847

8. Ma Z, Chen J, Lan F (2014) Biomechanical response and injury of occupant's pelvis in side impacts: effects of the femoral head and loading conditions. Journal of Mechanics in Medicine and Biology $14(06): 1440001$

9. Shibo G, Xuanhui Q, Xinbo H, Ting Z, Bohua D (2006) Powder injection molding of Ti-6Al-4V alloy. Journal of Materials Processing Technology 173 (3):310-314

10. Niinomi M (1998) Mechanical properties of biomedical titanium alloys. Materials Science and Engineering: A 243 (1-2):231-236

11. Carney K, Pereira JM, Revilock D, Matheny P (2009) Jet engine fan blade containment using an alternate geometry. International journal of impact engineering 36 (5):720-728

12. Morgan EF, Keaveny TM (2001) Dependence of yield strain of human trabecular bone on anatomic site. Journal of biomechanics 34 (5):569-577

13. Helgason B, Viceconti M, Rúnarsson TP, Brynjólfsson S (2008) On the mechanical stability of porous coated press fit titanium implants: a finite element study of a pushout test. Journal of biomechanics $41(8): 1675-1681$

14. Bayraktar HH, Morgan EF, Niebur GL, Morris GE, Wong EK, Keaveny TM (2004) Comparison of the elastic and yield properties of human femoral trabecular and cortical bone tissue. Journal of biomechanics $37(1): 27-35$

15. Sterling AJ, Torries B, Shamsaei N, Thompson SM, Seely DW (2016) Fatigue behavior and failure mechanisms of direct laser deposited Ti-6Al-4V. Materials Science and Engineering: A 655:100-112

16. Bobbili R, Ramakrishna B, Madhu V (2017) Dynamic compressive behavior and fracture modeling of Titanium alloy IMI 834. Journal of Alloys and Compounds 714:225-231

17. Li J, Han L, Zhang H, Zhao Z, Su X, Zhou J, Li C, Yin P, Hao M, Wang K (2019) Medial sustainable nail versus proximal femoral nail antirotation in treating AO/OTA 31-A2. 3 fractures: Finite element analysis and biomechanical evaluation. Injury 50 (3):648-656

18. Eberle S, Gerber C, Von Oldenburg G, Hungerer S, Augat P (2009) Type of hip fracture determines load share in intramedullary osteosynthesis. Clinical Orthopaedics and Related Research® 467 (8):19721980 
19. Nuño N, Amabili M, Groppetti R, Rossi A (2002) Static coefficient of friction between Ti-6Al-4V and PMMA for cemented hip and knee implants. Journal of Biomedical Materials Research: An Official Journal of The Society for Biomaterials and The Japanese Society for Biomaterials 59 (1):191-200

20. Wang L, Zhao F, Han J, Wang C, Fan Y (2012) Biomechanical study on proximal femoral nail antirotation (PFNA) for intertrochanteric fracture. Journal of Mechanics in Medicine and Biology 12 (04):1250075

21. Palm H, Jacobsen S, Sonne-Holm S, Gebuhr P, Group HFS (2007) Integrity of the lateral femoral wall in intertrochanteric hip fractures: an important predictor of a reoperation. Journal of Bone and Joint Surgery 89 (3):470-475

22. Haidukewych GJ (2009) Intertrochanteric fractures: ten tips to improve results. Journal of Bone and Joint Surgery 91 (3):712-719

23. Huang Y, Zhang C, Luo Y (2013) A comparative biomechanical study of proximal femoral nail (InterTAN) and proximal femoral nail antirotation for intertrochanteric fractures. International orthopaedics 37 (12):2465-2473

24. Khor F, Cronin DS, Watson B, Gierczycka D, Malcolm S (2018) Importance of asymmetry and anisotropy in predicting cortical bone response and fracture using human body model femur in threepoint bending and axial rotation. Journal of the Mechanical Behavior of Biomedical Materials 87:213-229

\section{Figures}




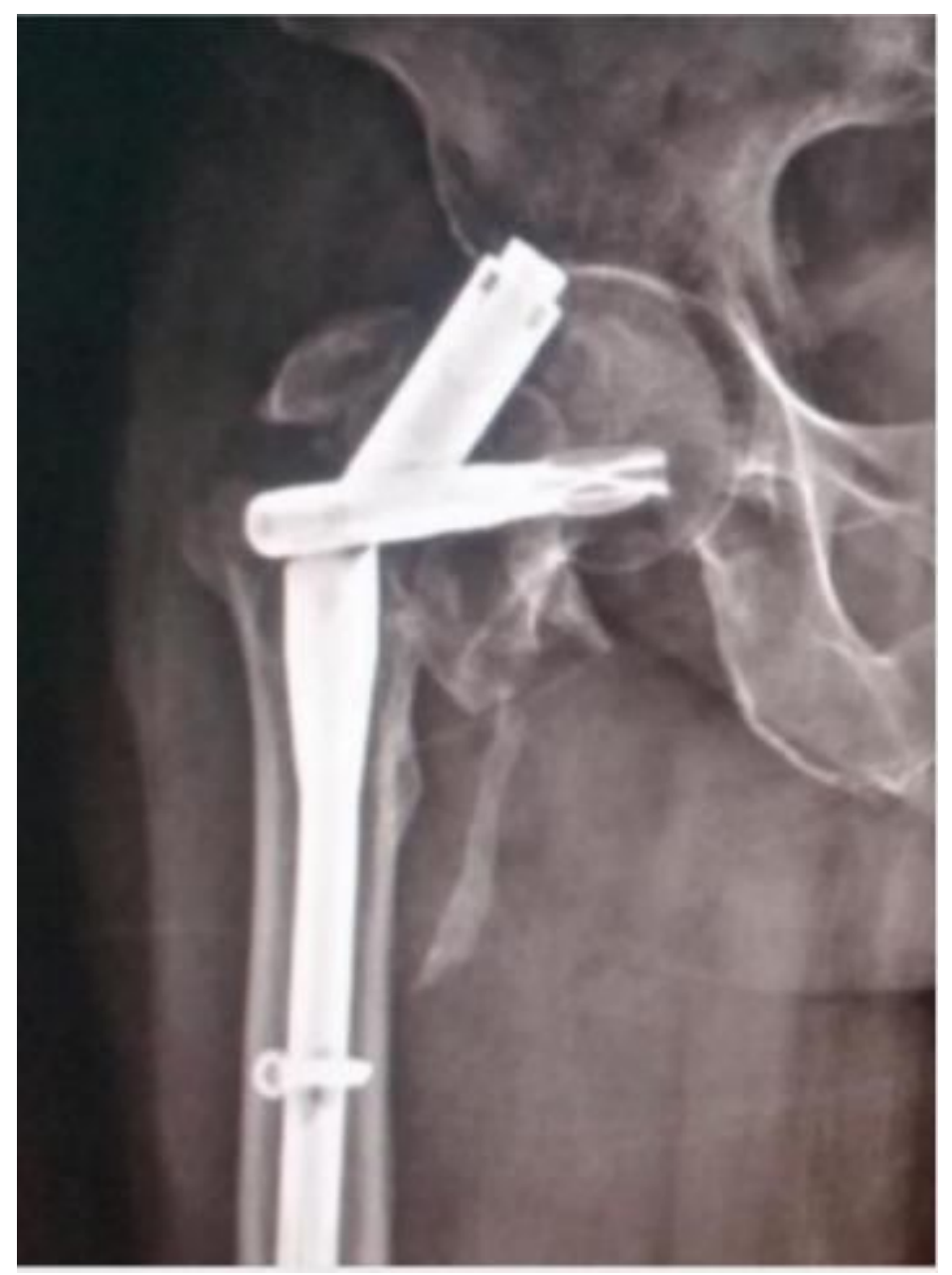

Figure 1

demonstrates a case of complication with PFNA nail fracture 


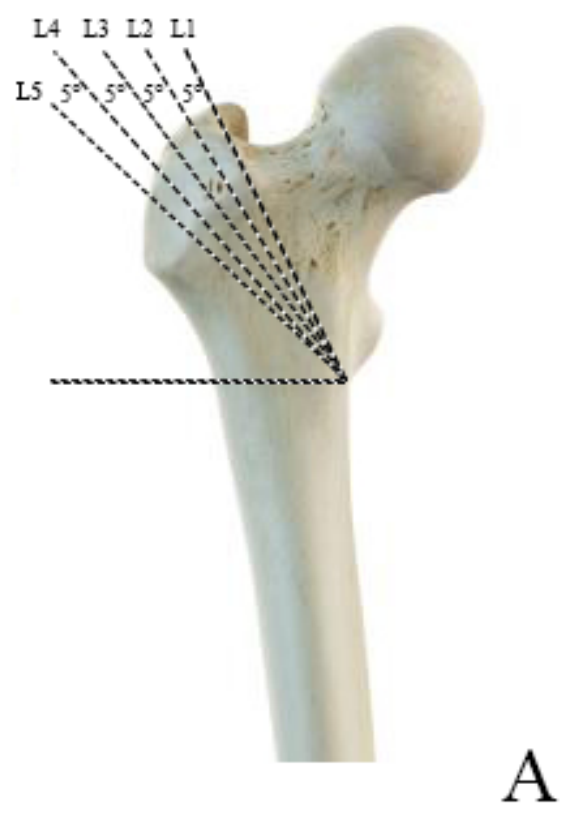

\section{Figure 2}

Lateral femoral wall thickness reflects the integrity and stability of the intertrochanteric fracture condition [5]. It was defined as the distance of a line started from a point 3-cm inferior to the innominate tubercle of the greater trochanter, angled at $135^{\circ}$ from the upward direction, and headed towards the fracture line on the coronal plane [6], as illustrated
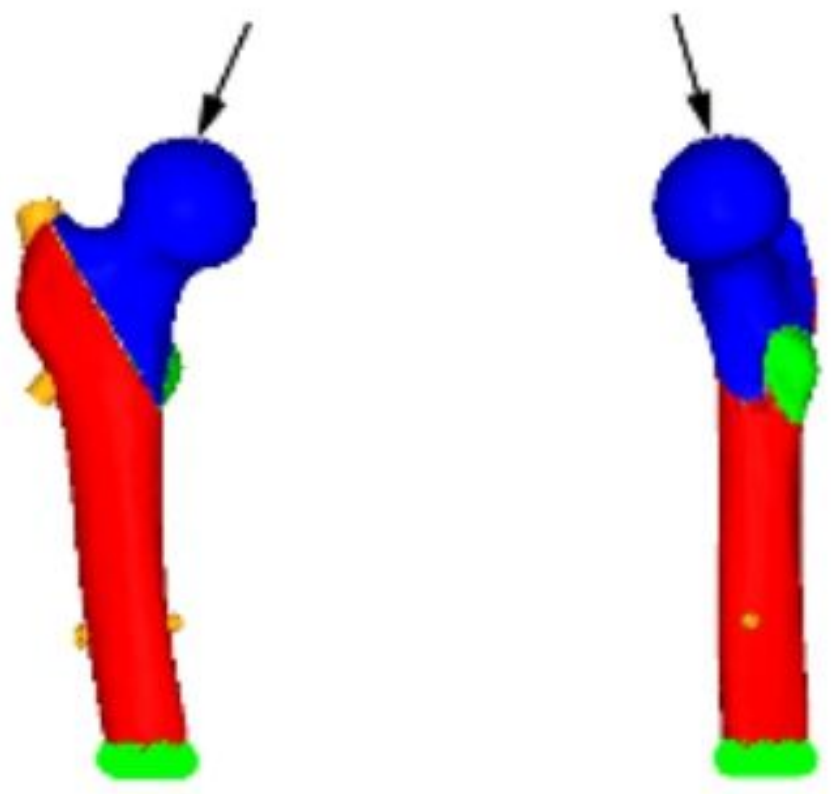

Figure 3 
The femoral head was loaded $2,100 \mathrm{~N}$ at $10^{\circ}$ lateral to the inferior axis on the frontal plane and $9^{\circ}$ posterior to the inferior axis on the sagittal plane [17], as demonstrated

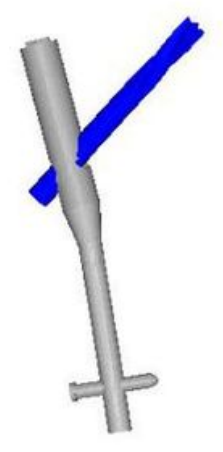

T1

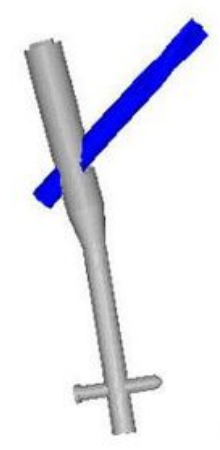

$\mathrm{T} 2$

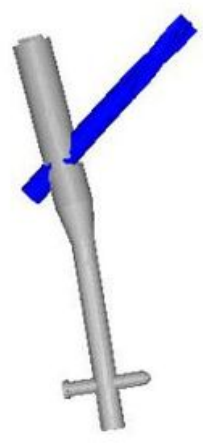

T3

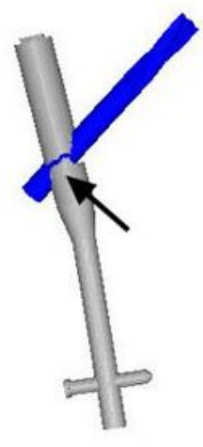

$\mathrm{T} 4$

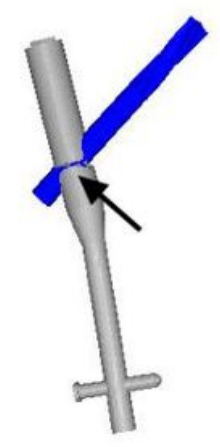

$\mathrm{T} 5$

\section{Figure 4}

As shown in Figure 4, implant failure of PNFA occurred at the sides of the proximal nail canal where the spiral blade passed through.

T1
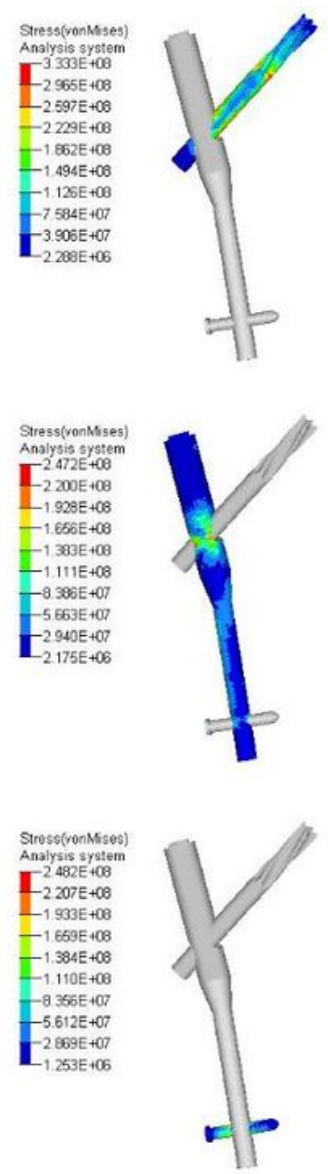

T2
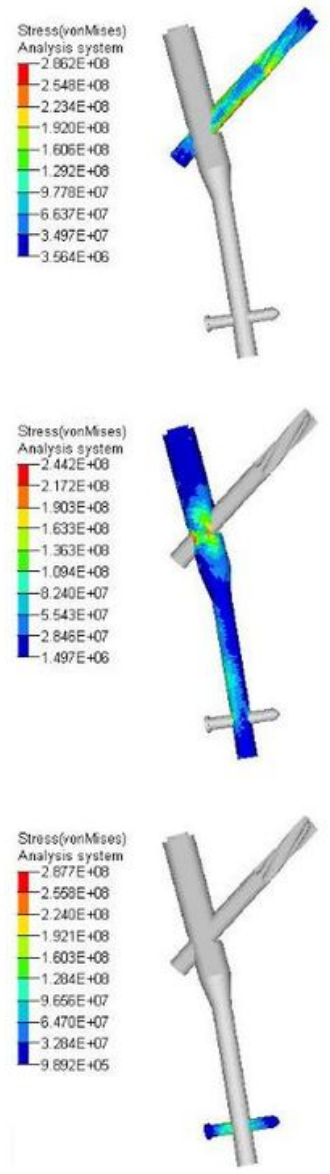

T3
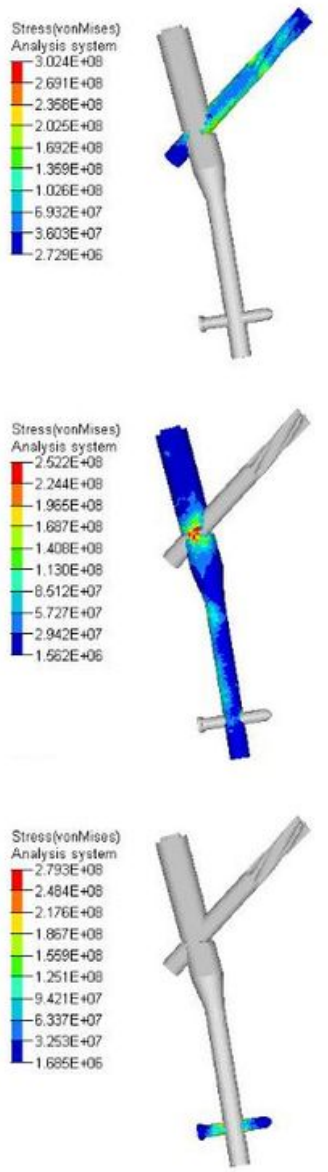

T4

T5
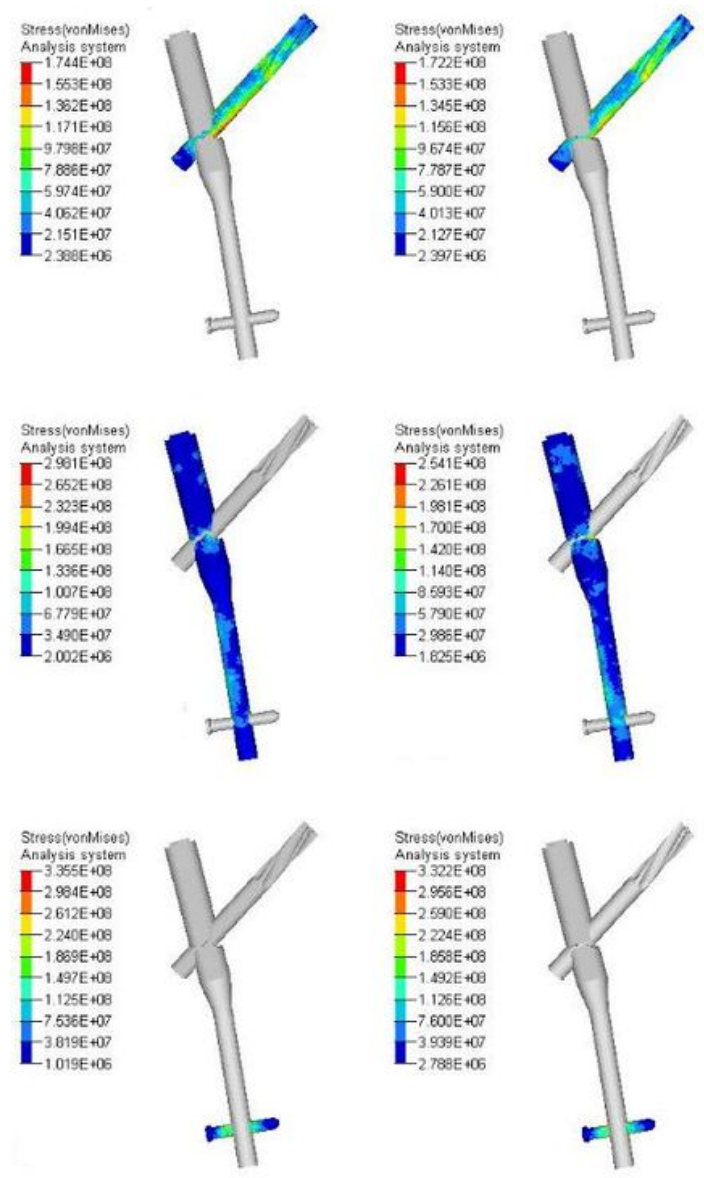

Figure 5 
For the PNFA nail, stress was concentrated at the proximal and distal canals which passed through with the spiral blade and locking screw, respectively

$\mathrm{T} 1$

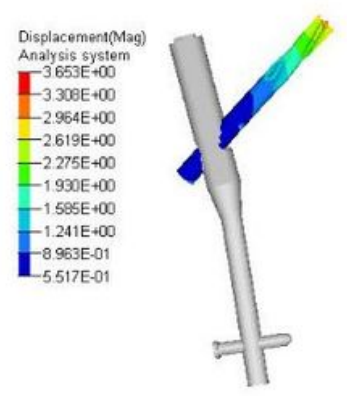

Displacement(Mag)
Analysis system Analysis syste

-7 $892 \mathrm{E}-01$

$-7.012 E-01$
$-6.133 E-01$

$-5.133 E-01$
$-5.253 E-01$

$-5.253 \mathrm{E}-01$
$-4.373 \mathrm{E}-01$

- $3.494 \mathrm{E}-01$

$-2614 \mathrm{E}-01$
$-1.734 \mathrm{E}-01$
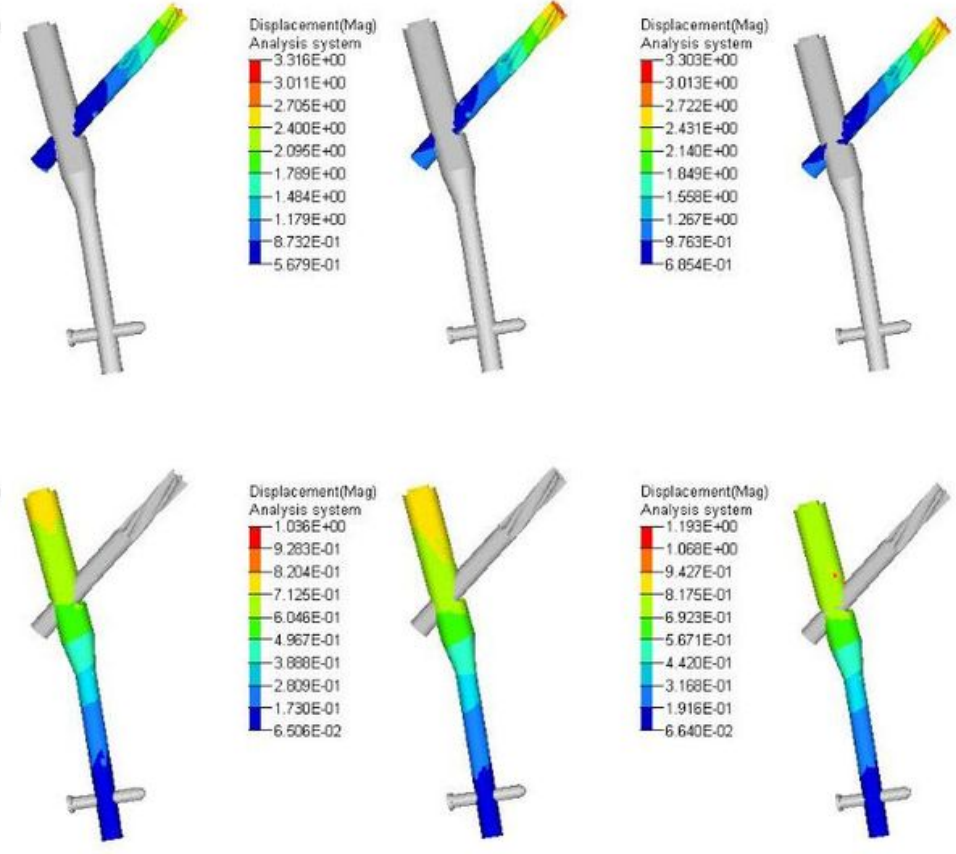

T3
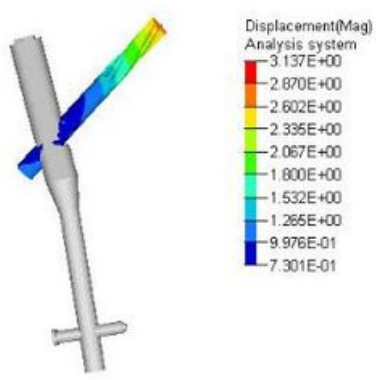

T4
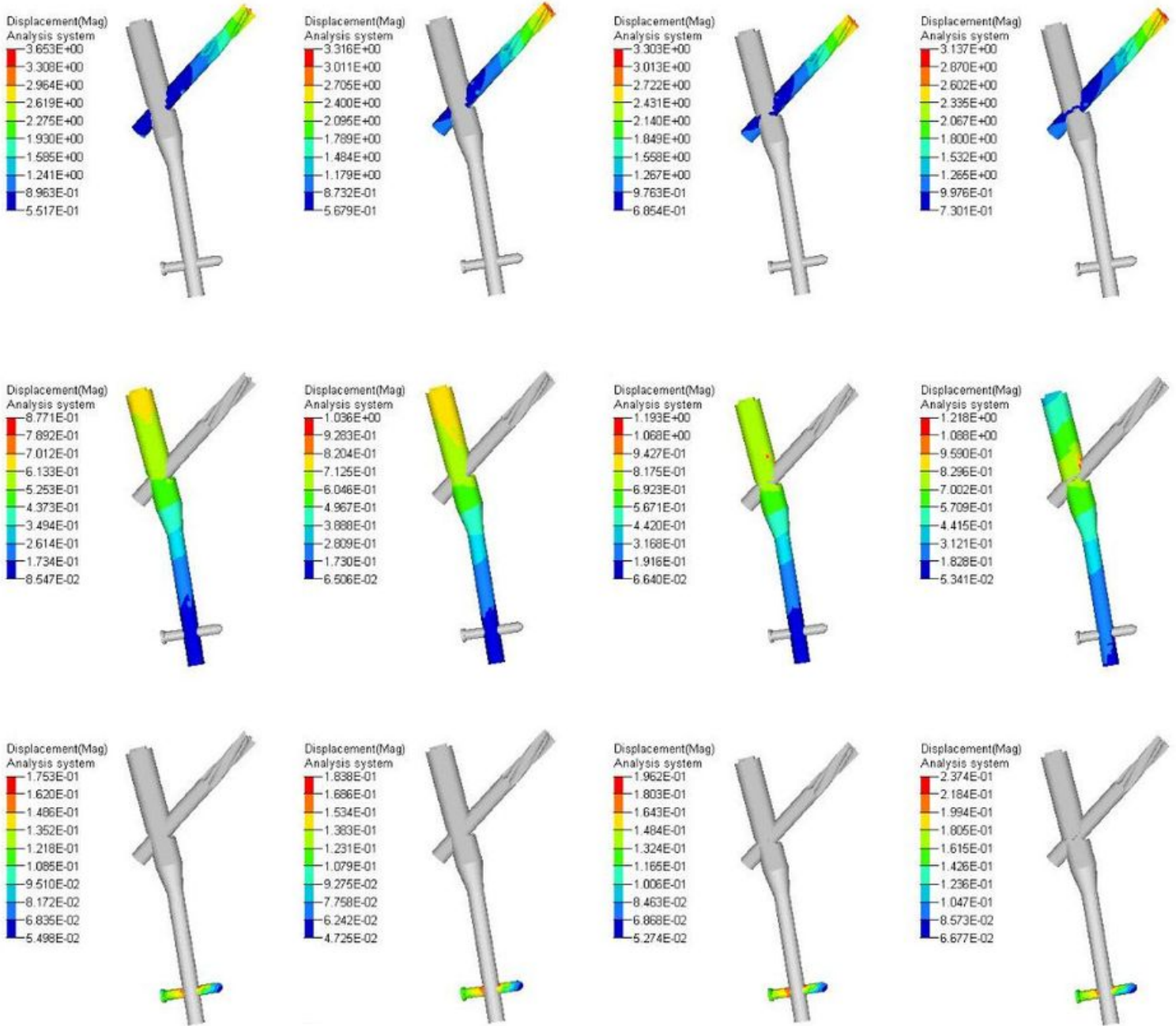

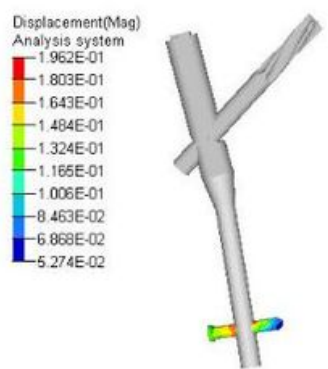

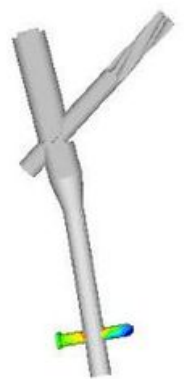
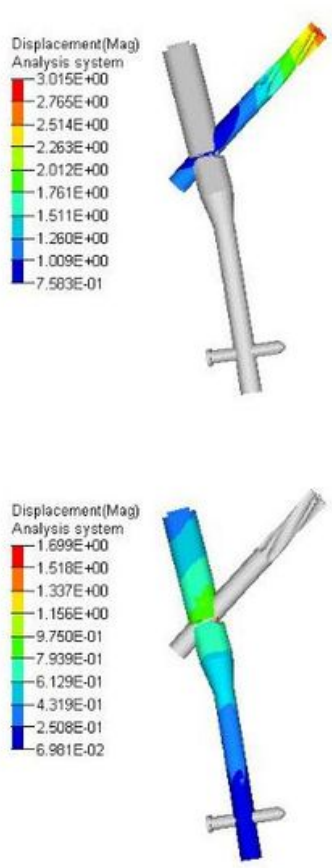

T5
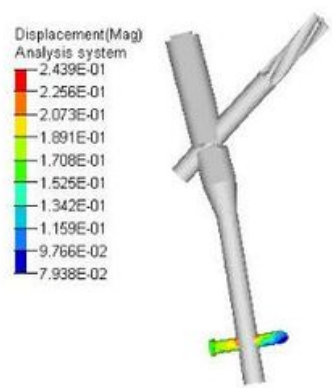

Figure 6

The maximum resultant displacement was presented at the proximal end of the nail before breakage and around the proximal nail canal after breakage 

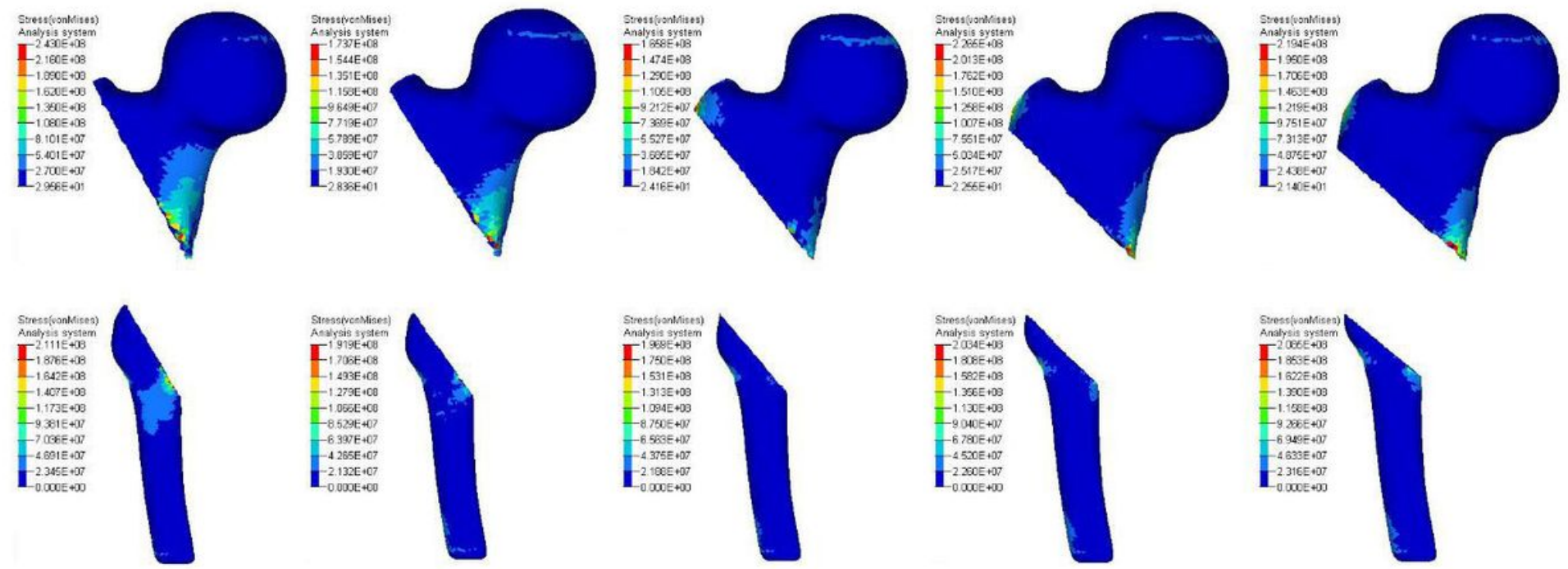

T1

T2

T3

T4

T5
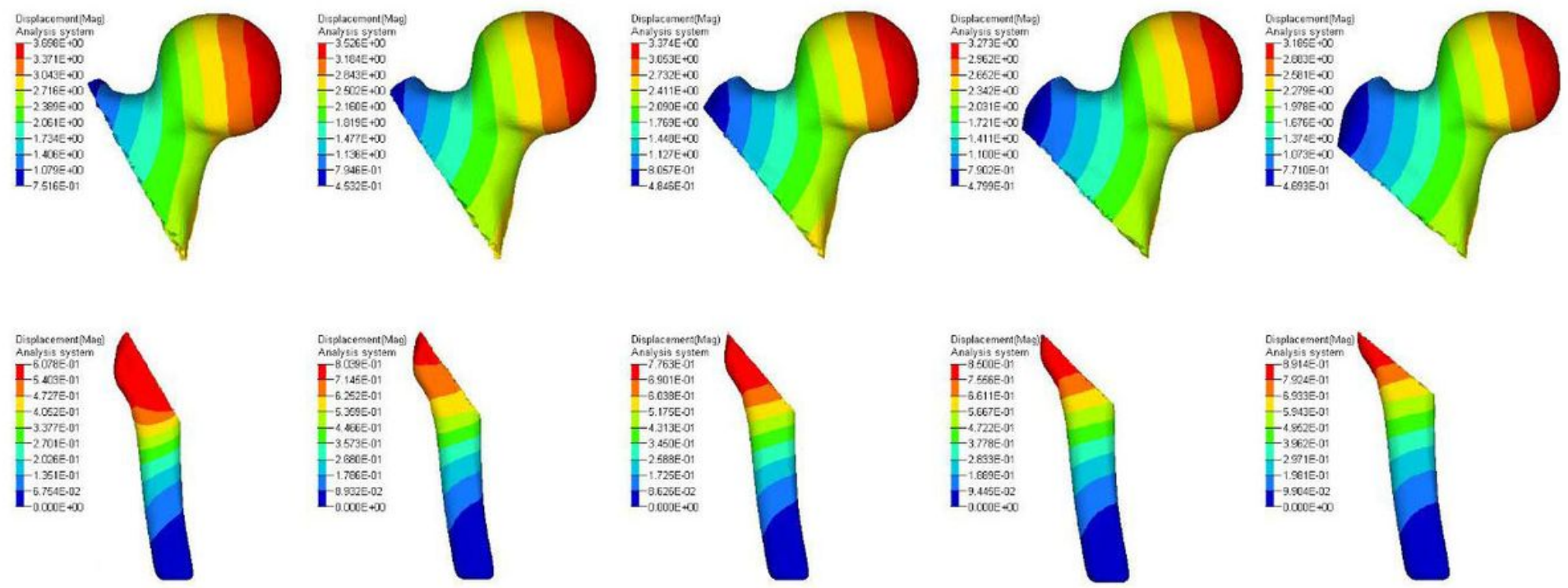

Figure 7

For T1 and T2, the lateral femoral wall thickness corresponded to a fracture line inside the entry point such that the stress was concentrated at the interoinferior cortex of the bone 